\title{
Mediastinoscopy-assisted esophagectomy for T2 middle and lower thoracic esophageal squamous cell carcinoma patients
}

Jun Wang, Ning Wei, Yimin Lu, Xiaoying Zhang and Nanqing Jiang*

\begin{abstract}
Background: We aimed to compare mediastinoscopy-assisted esophagectomy (MAE) with the Ivor Lewis procedure in T2 middle and lower thoracic esophageal carcinoma patients in fields of perioperative complications and overall survival (OS).

Methods: The clinical data of 112 T2 esophageal cancer patients who received MAE $(n=31)$ or Ivor Lewis procedure $(n=81)$ from January 2010 to December 2015 were retrospectively analyzed in propensity score analysis. Thirty-eight T2 esophageal cancer patients who underwent MAE $(n=19)$ and Ivor Lewis procedure $(n=19)$ were included in this study. The perioperative conditions and OS were analyzed.

Results: The MAE group showed shorter operation time (143.2 \pm 20.6 vs $176.8 \pm 31.1 \mathrm{~min}, P=0.001)$, less drainage in $24 \mathrm{~h}(119.2 \pm 235.1$ vs $626.3 \pm 396.3 \mathrm{~mL}, P<0.001)$, less retention time of thoracic tube $(27.8 \pm 24.0 \mathrm{vs} 101.2 \pm 54.6 \mathrm{~h}$, $P<0.001)$, and less hemorrhage during operation $(255.4 \pm 159.8$ vs $367.4 \pm 150.9 \mathrm{~mL}, P=0.059)$ compared with the Ivor Lewis group. Less dissected lymph nodes were detected in the MAE group $(12.2 \pm 5.4 \mathrm{vs} 16.8 \pm 5.8, P=0.044)$ than in the Ivor Lewis group, especially in the upper mediastinum (1.8 \pm 2.1 vs $3.5 \pm 2.3, P<0.001)$ and middle mediastinum $(2.5 \pm 2.0$ vs $5.3 \pm 3.2, P=0.027)$. The mean survival time was 59.1 and 53.3 months for the MAE group and Ivor Lewis group, respectively $(P=0.635)$. The results of Cox regression indicated that the nodal stage $(P=0.016)$ was an independent prognostic factor and the surgical method was not an independent prognostic factor for these patients $(P=0.290)$.

Conclusions: MAE procedure showed less surgical trauma compared with the Ivor Lewis procedure. The mediastinal lymphadenectomy of T2 esophageal carcinoma patients who underwent MAE was inferior to those who underwent Ivor Lewis procedure. The perioperative complications and OS of the MAE group were no worse than that of the Ivor Lewis group.
\end{abstract}

Keywords: Esophageal carcinoma, Ivor Lewis, Mediastinoscopy-assisted esophagectomy

\footnotetext{
* Correspondence: jiangnanqing8108@163.com

The First People's Hospital of Changzhou, The Third Affiliated Hospital of

Soochow University, 185 Juqian Road, Changzhou, Jiangsu Province 213003,

China
} 


\section{Background}

Esophageal carcinoma is a common malignancy with high morbidity and mortality worldwide [1-3]. Esophageal carcinoma was with about 0.48 million new cases and 0.44 million deaths in 2015 [4]. The morbidity of esophageal carcinoma is estimated to increase in the future [5]. China has the most esophageal carcinoma patients of the entire world [6]. The esophageal carcinoma is the sixth most common malignancy in China. And the mortality of esophageal carcinoma ranks fourth among all malignant tumors in China [6]. Esophageal carcinoma can be divided into two main subtypes: esophageal adenocarcinoma and esophageal squamous cell carcinoma (ESC). Most esophageal carcinoma patients in the western country suffered from esophageal adenocarcinoma [7]. The incidence of ESC was more than $90 \%$ in China [8]. The middle and lower thoracic esophageal carcinoma patients account for more than $80 \%$ of all esophageal carcinoma patients in China [7]. Esophagectomy is an important modality for ESC treatment. The controversies about the operative approach of esophagectomy still continue [9]. Both the Ivor Lewis esophagectomy (Ivor Lewis) and the mediastinoscopyassisted esophagectomy (MAE) have been performed in our department for a long time. MAE was reported by Buess and Becker in 1990 [10]. It is still controversial because of the insufficient mediastinal lymphadenectomy in MAE procedure [11]. Our previous study showed that MAE was suitable for T1 esophageal carcinoma patients and had an optimal longtime survival rate [12]. On this basis, MAE has been performed for esophageal carcinoma patients with preoperative stage T1 in our department since 2010. The potential differences between the preoperative $\mathrm{T}$ stage and the postoperative $\mathrm{T}$ stage cause some unexpected T2 patients received MAE in our department [13]. This study aimed to compare MAE with Ivor Lewis procedures in T2 esophageal carcinoma in terms of perioperative complications, postoperative pathological findings, and long-term survival rate.

\section{Methods}

From January 2010 to December 2015, T2 esophageal carcinoma patients confirmed by postoperative pathological examination, who underwent MAE or Ivor Lewis procedure in Changzhou First People's Hospital, were enrolled in this study.

The operations were carried out by the experienced surgeons with having performed more than $80 \mathrm{MAE}$ and more than 100 Ivor Lewis esophagectomies. Patients were included only if they satisfied the following criteria: (a) not suffered from other malignant diseases, (b) without history of gastric or esophageal surgery, (c) no major organ dysfunction, (d) Karnofsky Index score greater than or equal to 90, (e) no history of neoadjuvant chemotherapy or radiotherapy, (f) without distant metastasis, and (g) no history of endoscopic therapies. All patients underwent detailed history collection and physical examination preoperation. The pulmonary function tests, arterial blood gas analysis, serum biochemical indexes, coagulation indexes, and cardiac ultrasonography were performed for all patients. The diagnoses were all identified by endoscopy and biopsies. CT scans of the cervical region, thorax, and upper abdomen were obtained for all patients. This study was approved by the Clinical Research Ethics Committee of our hospital.

\section{Surgical procedure}

The MAE procedure was performed as previously described [12]. Patients were in the horizontal position. The cervical esophagus was dissected via the incision of anterior border of sternomastoid muscle (Fig. 1a). The electric coagulation/aspiration was used to dissect the thoracic esophagus along the esophagus bed (Fig. 1c, d). The titanium clips were used for dealing with esophageal artery (Fig. 1e, f). The mediastinal lymph nodes were resected through the use of coagulation/aspiration and biopsy forceps (Fig. 1g, h). The epigastria midline incision was adopted to dissect the stomach and perform the abdominal lymphadenectomy (Fig. 1b). Gastroesophagostomy was performed in the neck by using the hand-sewn method. The nasal feeding tube was placed.

The Ivor Lewis procedure was performed as described below. Patients were in the supine position to dissect the stomach and perform the abdominal lymphadenectomy via midline incision on the upper abdomen. Patients were changed to the left lateral position after abdominal surgery. A posterolateral incision in the fifth intercostal space was used to dissect thoracic esophagus and clear away the thoracic lymph nodes. Esophagogastric anastomosis was performed in the uppermost part of the thorax. Patients were changed to the supine position again to place the nasal feeding tube. The Ivor Lewis procedure and MAE were performed by the same team.

\section{Propensity score matching}

Propensity score matching was adopted to match subjects in the MAE and Ivor Lewis groups. The propensity scores were calculated by gender, age, tumor location, tumor length, histological grading, and nodal status. Using the nearest neighbor method, caliper value 0.01 , and 1:1 matching algorithm, 19 of 31 patients undergoing MAE and 19 of 81 patients undergoing the Ivor Lewis procedure were matched and analyzed.

\section{Pathological examination}

Pathological examination was performed to assess the tumor morphology, resection margin, histological grading, vessel invasion, tumor length, lymphatic metastasis, infiltration 

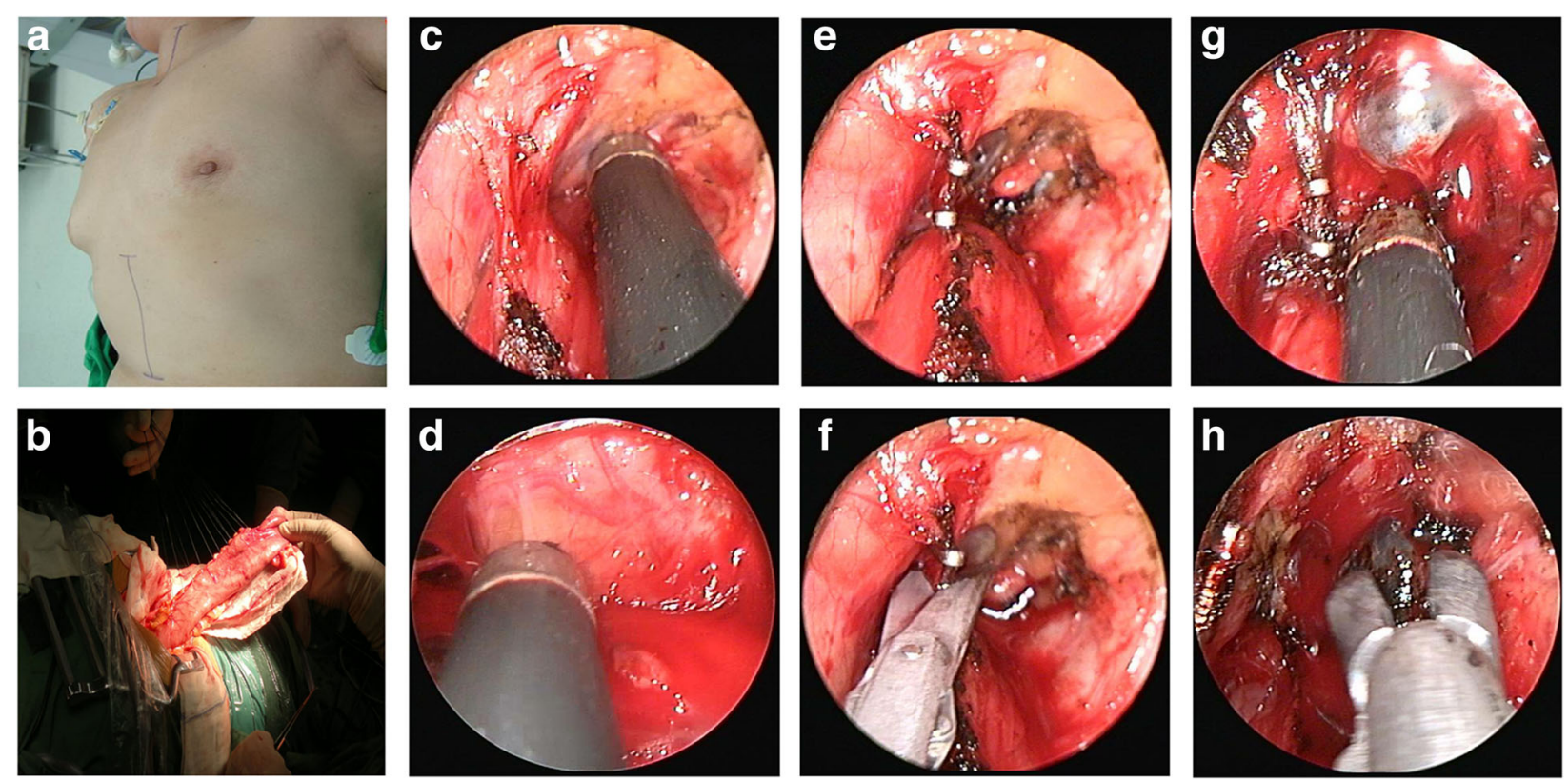

Fig. 1 This material was originally published in [Mediastinoscopy-assisted oesophagectomy in T1 oesophageal cancer patients with serious comorbidities: a 5-year long-term follow-up] by / edited by ([12] and Oxford University Press), and has been reproduced by permission of Oxford University Press [http://global.oup.com/academic]. a The patient positioning and surgical approach. b Gastric mobilization was performed via epigastria midline incision. $\mathbf{c}, \mathbf{d}$ The coagulation/aspiration was used to dissect the thoracic esophagus along the esophageal bed. $\mathbf{e}, \mathbf{f}$ The esophageal artery was handled by titanium clips under mediastinoscopy. $\mathbf{g}$ and $\mathbf{h}$ show the resection of mediastinal lymph nodes under mediastinoscopy

depth, and lymphatic metastasis. The eighth edition of the TNM classification for esophageal carcinoma maintained by the American Joint Committee on Cancer (AJCC) was adopted for the postoperative pathological staging.

\section{Statistical analysis}

We followed up these patients until October 2016 via rediagnosis, telephone calls, and letters. Data were expressed as mean \pm standard deviation. The main endpoint of this

Table 1 Patient characteristics and laboratory findings of the patients

\begin{tabular}{llll}
\hline & MAE & Ivor Lewis & $P$ \\
\hline Male/female & $12: 7$ & $13: 6$ & 0.732 \\
Median age, year & 62 & 62 & 0.237 \\
Mean age, year & $63.1 \pm 7.1$ & $63.2 \pm 4.8$ & 0.794 \\
Smoker/nonsmoker & $7: 12$ & $8: 11$ & 0.740 \\
FEV1.0, L & $2.38 \pm 0.48$ & $2.49 \pm 0.43$ & 0.465 \\
FEV1.0/FVC (\%) & $81.77 \pm 11.18$ & $84.37 \pm 8.28$ & 0.426 \\
PaO $_{2}, \mathrm{mmHg}$ & $84.75 \pm 10.10$ & $86.58 \pm 9.13$ & 0.587 \\
Creatinine, $\mu$ mol L $^{-1}$ & $80.80 \pm 12.59$ & $84.18 \pm 16.49$ & 0.486 \\
Blood glucose, mmol L & $5.13 \pm 0.88$ & $5.43 \pm 1.56$ & 0.466 \\
EF (\%) & $60.1 \pm 3.3$ & $58.8 \pm 2.9$ & 0.488 \\
PT, s & $11.20 \pm 0.84$ & $11.42 \pm 0.86$ & 0.436 \\
\hline FEV1.0 forced expirary & &
\end{tabular}

FEV1.0 forced expiratory volume in one second, $F V C$ forced expiratory volume, $E F$ ejection fraction, $P T$ prothrombin time study was the OS. A statistical software package SPSS 22.0 (SPSS, Inc. IL, USA) was used. Continuous variables were compared using $T$ tests. Categorical variables were compared using the chi-square test. Fisher' exact test was adopted if necessary. The OS was analyzed by the KaplanMeier method and Cox multivariate regression analysis. Statistical significance was defined as $P<0.05$.

\section{Results}

\section{Patient characteristics and laboratory findings}

Thirty-eight T2 esophageal carcinoma patients who underwent $\operatorname{MAE}(n=19)$ and the Ivor Lewis $(n=19)$ procedure in our department were included in this nested retrospective study. The preoperative clinical data of these patients are given in Table 1. No statistically significant difference was found between the groups in terms of gender, age, tobacco use, forced expiratory volume in one second (FEV1.0), FEV1.0/forced vital capacity (FVC), $\mathrm{PaO}_{2}$, creatinine, blood glucose, ejection fraction (EF), or prothrombin time before operation.

\section{Perioperative conditions}

Postoperative complications were assessed as described before [14]. The conditions during perioperative period of the patients are given in Table 2. No statistically significant difference was found between the groups in terms of postoperative hospital stay, anastomotic fistula, pulmonary infection, laryngeal recurrent nerve injury, 
Table 2 Perioperative conditions of the patients

\begin{tabular}{llll}
\hline & MAE & Ivor Lewis & $P$ \\
\hline Operation time, min & $143.2 \pm 20.6$ & $176.8 \pm 31.1$ & 0.001 \\
Hemorrhage in operation, $\mathrm{mL}$ & $255.4 \pm 159.8$ & $367.4 \pm 150.9$ & 0.059 \\
Drainage in $24 \mathrm{~h}, \mathrm{~mL}$ & $119.2 \pm 235.1$ & $626.3 \pm 396.3$ & $<0.001$ \\
$\begin{array}{l}\text { Retention time of the } \\
\text { thoracic tube, } \mathrm{h}\end{array}$ & $27.8 \pm 24.0$ & $101.2 \pm 54.6$ & $<0.001$ \\
$\begin{array}{l}\text { Postoperative hospital stay, days } \\
\text { Anastomotic fistula }\end{array}$ & $11.1 \pm 7.2$ & $11.3 \pm 6.5$ & 0.998 \\
Pulmonary infection & 0 & 1 & 0.290 \\
Laryngeal recurrent nerve & 1 & 1 & $>0.99$ \\
damage & & 0 & $>0.99$ \\
Arrhythmia & 2 & 0 & 0.486 \\
Gastric retention & 0 & 3 & 0.230 \\
Chylothorax & 1 & 1 & $>0.99$ \\
Incision infection & 3 & 1 & 0.640 \\
\hline
\end{tabular}

arrhythmia, chylothorax, and incision infection. The MAE group showed shorter operation time (143.2 \pm 20.6 vs $176.8 \pm 31.1 \mathrm{~min}, P=0.001)$ and less volume of drainage in $24 \mathrm{~h}(119.2 \pm 235.1$ vs $626.3 \pm 396.3 \mathrm{~mL}, P<0.001)$ compared with the Ivor Lewis group. The retention time of the thoracic tube also significantly reduced in the MAE group $(27.8 \pm 24.0 \mathrm{~h}, P<0.001)$ than in the Ivor Lewis group $(101.2 \pm 54.6 \mathrm{~h})$. The MAE group also showed less hemorrhage during operation (255.4 \pm 159.8 vs $367.4 \pm 150.9 \mathrm{~mL}, P=0.059)$.

\section{Postoperative pathological findings}

The eighth edition of the AJCC classification for esophageal carcinoma was adopted for the postoperative pathological staging. The postoperative pathological findings are listed in Table 3. No significant difference was found in tumor location, tumor morphology, resection margin, histological grading, vessel invasion, tumor length, nodal status, TNM stage, and number of positive lymph nodes. The MAE group showed less dissected lymph nodes $(12.2 \pm 5.4$ vs $16.8 \pm 5.8, P=0.044)$ than the Ivor Lewis group, especially in the upper mediastinum $(1.8 \pm 2.1$ vs $3.5 \pm 2.3, P<0.001)$ and middle mediastinum $(2.5 \pm 2.0$ vs $5.3 \pm 3.2, P=0.027$ ).

\section{Long-term outcomes}

We followed up these patients until death or October 2016. The median follow-up time was 31.0 months (780). The mean survival time was 59.1 months [95\% confidence interval (CI) 45.2-72.9] for the MAE group and 53.3 months (95\% CI 38.2-68.4) for the Ivor Lewis group (Fig. 2). No significant difference was found in the OS between the two groups $(P=0.635)$. Results of the multivariate analysis by Cox regression demonstrated that the nodal stage was an independent prognostic
Table 3 Postoperative pathological findings

\begin{tabular}{llll}
\hline & MAE & Ivor Lewis & $P$ \\
\hline Tumor location (middle/lower) & $13: 6$ & $15: 4$ & 0.461 \\
Tumor morphology (ulcer/ & $13: 2: 4$ & $13: 4: 2$ & 0.513 \\
medullary/masses) & & & \\
Resection margin (R0:R1) & $19: 0$ & $18: 1$ & $>0.99$ \\
Histological grading (G2:G3) & $12: 7$ & $10: 9$ & 0.511 \\
Vessel invasion & 1 & 2 & 0.547 \\
Tumor length & $2.8 \pm 0.7$ & $2.9 \pm 0.8$ & 0.665 \\
Nodal status (NO:N1:N2:N3) & $13: 4: 2: 0$ & $14: 2: 2: 1$ & 0.636 \\
TNM stage (Ila: Illa: IIIb:IVa) & $13: 4: 2: 0$ & $14: 2: 2: 1$ & 0.636 \\
Number of positive lymph nodes & $0.8 \pm 1.6$ & $1.0 \pm 2.4$ & 0.281 \\
Dissected lymph node number & $12.2 \pm 5.4$ & $16.8 \pm 5.8$ & 0.044 \\
$\quad$ Upper mediastinum & $1.8 \pm 2.1$ & $3.5 \pm 2.3$ & $<0.001$ \\
$\quad$ Middle mediastinum & $2.5 \pm 2.0$ & $5.3 \pm 3.2$ & 0.027 \\
$\quad$ Lower mediastinum & $2.1 \pm 1.9$ & $1.9 \pm 1.4$ & 0.850 \\
Abdominal & $5.8 \pm 2.9$ & $5.6 \pm 3.0$ & 0.687 \\
Postoperative radiotherapy & 6 & 8 & 0.501 \\
Postoperative chemotherapy & 9 & 8 & 0.744 \\
\hline
\end{tabular}

factor for these patients $(P=0.016)$. The surgical method was not an independent prognostic factor for these patients $(P=0.290)$. Postoperative radiotherapy $(P=0.595)$ was not an independent prognostic factor for these patients, as well as the postoperative chemotherapy $(P=$ 0.731) (Table 4).

\section{Discussion}

ESC occurs with high morbidity and mortality in China [3]. Esophageal resection is regarded as the cornerstone of treatment for esophageal carcinoma patients to date. Transthoracic esophagectomy is a complex procedure

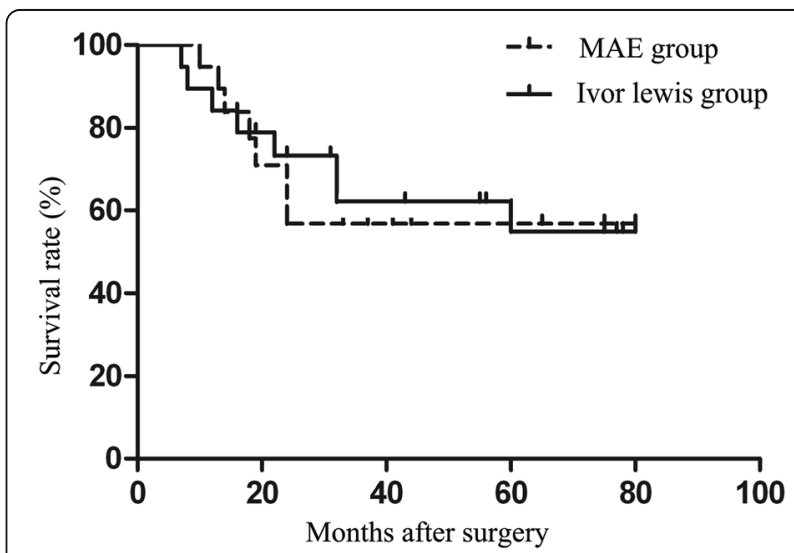

Fig. 2 Overall survival curves after MAE and Ivor Lewis procedure. The mean survival time was 59.1 months [95\% confidence interval (CI) 45.2-72.9] for the MAE group and 53.3 months (95\% Cl 38.268.4) for the Ivor Lewis group. No significant difference was found in the long-term survival rate between the two groups $(P=0.635)$ 
Table 4 Results of the Cox regression model for the analysis of prognosis

\begin{tabular}{lllll}
\hline & $P$ & HR & \multicolumn{2}{l}{$95.0 \%$ Cl for Exp(B) } \\
\cline { 4 - 5 } & & & Lower & Upper \\
\hline Age & 0.454 & 0.970 & 0.897 & 1.050 \\
Tumor location & 0.505 & 0.725 & 0.281 & 1.868 \\
Tumor type & 0.260 & 0.749 & 0.454 & 1.237 \\
Tumor differentiation & 0.354 & 0.537 & 0.148 & 1.951 \\
Vessel invasion & 0.592 & 0.588 & 0.084 & 4.012 \\
Nodal stage & 0.016 & 3.155 & 1.548 & 5.383 \\
Tumor length & 0.379 & 0.728 & 0.369 & 1.477 \\
Postoperative & 0.595 & 1.443 & 0.373 & 5.582 \\
radiotherapy & & & & \\
Postoperative & 0.731 & 1.189 & 0.444 & 3.181 \\
chemotherapy & & & & \\
MAE/lvor Lewis & 0.290 & 0.579 & 0.377 & 5.549 \\
\hline
\end{tabular}

and associated with high surgical risk [15]. Thoracotomy can be avoided in MAE. However, MAE is still not widely acceptable because of the controversy about lymphadenectomy. Our previous study showed that MAE for patients with T1 esophageal carcinoma was safe and had a satisfied long-term survival rate [12]. However, more studies are needed for advanced esophageal carcinoma patients. This nested case-control study explored MAE versus Ivor Lewis procedure for T2 middle and lower thoracic esophageal carcinoma patients. The complications and outcomes of Ivor Lewis and MAE groups were retrospectively analyzed.

The MAE group was advantageous over the Ivor Lewis group in terms of shorter operation time. In the MAE procedure, esophagectomy using mediastinoscopy and gastric mobilization via upper abdominal incision could go on simultaneously [16]. This method greatly shortened the operation time. Changing positions were necessary for patients undergoing the Ivor Lewis procedure. And the abdominal and thoracic regions cannot be operated at the same time.

Opening thorax could be avoided in MAE. If the mediastinal pleura could maintain integrity during operation, the thoracic tube was not used in MAE. The thoracic tube is routinely used in the Ivor Lewis procedure. This procedure shortened the retention time of the thoracic tube in the MAE group. Complete mediastinal pleura could also limit bleeding by pressing, thereby greatly reducing the volume of drainage in $24 \mathrm{~h}$. A previous study showed that the lung function after operation could benefit from protection of the integrity of pleura [17]. The MAE group also showed less hemorrhage during operation compared with the Ivor Lewis group, although the result was not statistically significant $(255.4 \pm 159.8$ vs $367.4 \pm 150.9 \mathrm{~mL}, P=0.059)$. These results showed that the MAE procedure had less surgical trauma compared with the Ivor Lewis procedure.

In the present study, the Ivor Lewis group also showed more patients with gastric retention compared with the MAE group, although no statistically significant difference was found (Table 2). We fixed the intrathoracic stomach between the mediastinal pleura in MAE. The intrathoracic stomach is fixed to the right thorax in the Ivor Lewis procedure. Right intrathoracic stomach may reduce the stenosis of the stomach [18]. And right intrathoracic stomach may also lead to inappropriate angle between the pylorus and the stomach. This physical factor may increase the risk of delayed gastric emptying.

The result showed that the perioperative conditions of MAE for T2 esophageal carcinoma patients were acceptable and similar to or even superior to those of the Ivor Lewis procedure in some areas. MAE could be performed safely for T2 esophageal carcinoma patients.

Lymph node metastasis is an important factor affecting the OS of esophageal carcinoma patients. According to the TNM classification for esophageal carcinoma, 15 lymph nodes in every surgical specimen are required for reliable nodal status. In the present study, more dissected lymph nodes were found in the Ivor Lewis group than in the MAE group. Inadequate lymphadenectomy was found in the MAE group. A potential risk of tumor under-staging might be resulted in the MAE group. In MAE, the operation field in the mediastinum was restricted, and lymphadenectomy for the thorax was difficult [12]. In the Ivor Lewis procedure, good exposure of the thorax was beneficial for lymph node resections. The lymphadenectomy is a major disadvantage of MAE. This shortage could be covered by ameliorating the surgical technique and improving the surgical instruments $[11,19]$.

It was reported that more number of resected lymph nodes were correlated positively with survival time [20]. However, some studies reported that survival time was not able to benefit from radical lymphadenectomy [21]. The Kaplan-Meier method showed that there was no significant difference in the long-term survival rate between the groups $(P=0.635)$. Cox regression also showed that the surgical method was not an independent prognostic factor for these patients $(P=0.290)$. Our results showed that the long-term survival rate for patients with T2 esophageal carcinoma who received MAE was acceptable. Previous study showed that tumor length influenced the longtime survival rate [22], while the Cox regression showed that the tumor length was not an independent prognostic factor for patients in this study. This may result from the small sample size of this study.

Transthoracic esophagectomy is involved with high surgical risk. Previous study showed that the perioperative 
mortality of transthoracic esophagectomy was about 2-3\% [15]. It was reported that more than one-third patients who underwent transthoracic esophagectomy had perioperative complication [23]. Our result showed that both the long-term survival rate and the perioperative complications of MAE for T2 esophageal cancer patients were acceptable. MAE may provide a surgical option for T2 esophageal cancer patients, especially for those who cannot tolerate transthoracic esophagectomy. This retrospective nested case-control study was performed at a single center. Hence, randomized, controlled, multicenter clinical trials are needed to confirm the findings.

\section{Conclusion}

MAE procedure showed less surgical trauma compared with the Ivor Lewis procedure. The mediastinal lymphadenectomy of patients with T2 esophageal cancer who underwent MAE was inferior to those who underwent Ivor Lewis procedure. The perioperative complications and OS of MAE group were no worse than the Ivor Lewis group.

\section{Abbreviations}

AJCC: American Joint Committee on Cancer; EF: Ejection fractions; ESC: Esophageal squamous cell carcinoma; FEV: Forced expiratory volume; FVC: Forced vital capacity; MAE: Mediastinoscopy-assisted esophagectomy; OS: Overall survival

\section{Acknowledgements}

Not applicable.

\section{Funding}

This work was supported by the Foundation of Changzhou Educational Committee [QN201302].

\section{Availability of data and materials}

The datasets generated during the current study are available from the corresponding author on a reasonable request.

\section{Authors' contributions}

JW and NJ carried out the studies, participated in collecting the data, and drafted the manuscript. XZ and NW performed the statistical analysis and participated in its design. NW and YL helped to draft the manuscript. All authors read and approved the final manuscript.

\section{Ethics approval and consent to participate}

This study was approved by the Clinical Research Ethics Committee of our hospital. All participants provided written informed consent.

\section{Consent for publication}

All participants provided consent for publication.

\section{Competing interests}

The authors declare that they have no competing interests.

\section{Publisher's Note}

Springer Nature remains neutral with regard to jurisdictional claims in published maps and institutional affiliations.

Received: 13 September 2017 Accepted: 6 March 2018

Published online: 16 March 2018

\section{References}

1. Siegel RL, Miller KD, Jemal A. Cancer statistics, 2017. CA Cancer J Clin. 2017; 67:7-30.
2. Chen W, Zheng R, Zeng H, Zhang S, He J. Annual report on status of cancer in China, 2011. Chin J Cancer Res. 2015;27:2-12.

3. Chen WQ, Zheng RS, Zhang SW, Zeng HM, Zou XN, He J. Analysis of cancer incidence and mortality in elderly population in China, 2013. Zhonghua Zhong Liu Za Zhi. 2017;39:60-6.

4. Global Burden of Disease Cancer C, Fitzmaurice C, Allen C, Barber RM, Barregard L, Bhutta ZA, et al. Global, regional, and national cancer incidence, mortality, years of life lost, years lived with disability, and disability-adjusted life-years for 32 cancer groups, 1990 to 2015: a systematic analysis for the global burden of disease study. JAMA Oncol. 2017;3:524-48.

5. Napier KJ, Scheerer M, Misra S. Esophageal cancer: a review of epidemiology, pathogenesis, staging workup and treatment modalities. World I Gastrointest Oncol. 2014;6:112-20.

6. Chen WQ, Li H, Sun KX, Zheng RS, Zhang SW, Zeng HM, et al. Report of cancer incidence and mortality in China, 2014. Zhonghua Zhong Liu Za Zhi. 2018;40:5-13.

7. Zeng H, Zheng R, Zhang S, Zuo T, Xia C, Zou X, et al. Esophageal cancer statistics in China, 2011: estimates based on 177 cancer registries. Thorac Cancer. 2016;7:232-7.

8. Liang H, Fan JH, Qiao YL. Epidemiology, etiology, and prevention of esophageal squamous cell carcinoma in China. Cancer Biol Med. 2017;14:33-41.

9. Noordman BJ, Wijnhoven BP, van Lanschot JJ. Optimal surgical approach for esophageal cancer in the era of minimally invasive esophagectomy and neoadjuvant therapy. Dis Esophagus. 2016;29:773-9.

10. Buess $\mathrm{G}$, Becker HD. Minimally invasive surgery in tumors of the esophagus. Langenbecks Arch Chir Suppl II Verh Dtsch Ges Chir. 1990;1 18:1355-60.

11. Fujiwara H, Shiozaki A, Konishi H, Otsuji E. Mediastinoscope and laparoscope-assisted esophagectomy. J Vis Surg. 2016;2:125.

12. Wang J, Jiang NQ, Jiang B, Wang ZL, Zhang XY. Mediastinoscopy-assisted oesophagectomy in T1 oesophageal cancer patients with serious comorbidities: a 5-year long-term follow-up. Interact Cardiovasc Thorac Surg. 2015;20:477-81.

13. Smith BR, Chang KJ, Lee JG, Nguyen NT. Staging accuracy of endoscopic ultrasound based on pathologic analysis after minimally invasive esophagectomy. Am Surg. 2010;76:1228-31.

14. Lerut T, Moons J, Coosemans W, Van Raemdonck D, De Leyn P, Decaluwe $\mathrm{H}$, et al. Postoperative complications after transthoracic esophagectomy for cancer of the esophagus and gastroesophageal junction are correlated with early cancer recurrence: role of systematic grading of complications using the modified Clavien classification. Ann Surg. 2009;250:798-807.

15. Raymond DP, Seder CW, Wright CD, Magee MJ, Kosinski AS, Cassivi SD, et al. Predictors of major morbidity or mortality after resection for esophageal cancer: a Society of Thoracic Surgeons General Thoracic Surgery Database risk adjustment model. Ann Thorac Surg. 2016;102:207-14.

16. Feng $M X$, Wang $H$, Zhang $Y$, Tan $\sqcup$, Xu ZL, Qun W. Minimally invasive esophagectomy for esophageal squamous cell carcinoma: a case-control study of thoracoscope versus mediastinoscope assistance. Surg Endosc. 2012;26:1573-8.

17. Uzun A, Yener AU, Kocabeyoglu S, Cicek OF, Yasar E, Yener O, et al. Effects of pleural opening on respiratory function tests in cardiac surgery: a prospective study. Eur Rev Med Pharmacol Sci. 2013;17:2310-7.

18. Yu Y, Wang Z, Liu XY, Zhu XF, Chen QF. Therapeutic efficacy comparison of two surgical procedures to treat middle thoracic esophageal carcinoma. World J Surg. 2009;34:272-6.

19. Fujiwara H, Shiozaki A, Konishi H, Kosuga T, Komatsu S, Ichikawa D, et al. Single-port mediastinoscopic lymphadenectomy along the left recurrent laryngeal nerve. Ann Thorac Surg. 2015;100:1115-7.

20. Peyre CG, Hagen JA, DeMeester SR, Altorki NK, Ancona E, Griffin SM, et al. The number of lymph nodes removed predicts survival in esophageal cancer: an international study on the impact of extent of surgical resection. Ann Surg. 2008;248:549-56.

21. Chang AC, Ji H, Birkmeyer NJ, Orringer MB, Birkmeyer JD. Outcomes after transhiatal and transthoracic esophagectomy for cancer. Ann Thorac Surg. 2008:85:424-9.

22. Lin CS, Liu CY, Cheng CT, Tsai YC, Chiou LW, Lee MY, et al. Prognostic role of initial pan-endoscopic tumor length at diagnosis in operable esophageal squamous cell carcinoma undergoing esophagectomy with or without neoadjuvant concurrent chemoradiotherapy. J Thorac Dis. 2017;9:3193-207.

23. Bhagat R, Bronsert MR, Juarez-Colunga E, Weyant MJ, Mitchell JD, Glebova NO, et al. Postoperative complications drive unplanned readmissions after esophagectomy for cancer. Ann Thorac Surg. 2018; https://doi.org/10.1016/j. athoracsur.2017.12.024. 